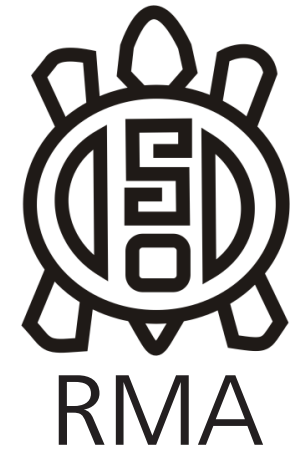

Dossier

\title{
Estrategias de producción de artefactos en la Unidad 4, -Holoceno medio- del sitio Cueva 1 de Cerro Tres Tetas
}

\author{
Artifact production strategies in Unit 4 -mid Holocene- in Cerro \\ Tres Tetas Cueva 1 site
}

Manuel Enrique Cueto*, Andrés Iparraguirre**, Rafael Sebastián Paunero**

* CONICET, División Arqueología, FCNyM,- Universidad Nacional de La Plata, Argentina. E-mail: manuelcueto@fcnym.unlp.edu.ar ** División Arqueología, FCNyM - Universidad Nacional de La Plata, Argentina. E-mail: iparraguirreandres@gmail.com; rpaunero@fcnym.unlp.edu.ar

\begin{abstract}
Resumen
En este artículo estudiamos la tecnología lítica del componente asignable al Holoceno medio, del sitio Cerro Tres Tetas 1 emplazado en la Meseta Central de Santa Cruz, Argentina. Las tendencias regionales para el periodo exponen el empleo de técnicas de talla y reducción bifacial, mayor densidad artefactual y el incremento en la producción de láminas, respecto de momentos previos. El objetivo es analizar las estrategias de producción seguidas en la fabricación de los instrumentos. Se aborda el conjunto desde una perspectiva tecno morfológica orientada a identificar relaciones entre los artefactos, para evaluar las actividades de talla, las técnicas y las decisiones involucradas en los procesos de producción. Los resultados indican la selección de materias primas locales, el empleo de técnicas de reducción estandarizada y no estandarizada de núcleos, el aumento en la elección de soportes laminares para la manufactura de instrumentos y el predominio de secuencias de producción con baja inversión de trabajo.
\end{abstract}

Palabras clave: Tecnología lítica; Estrategias de producción; Holoceno medio; Meseta Central; Patagonia.

\begin{abstract}
The present article studies lithic technology in components assigned to the Middle Holocene from Cerro Tres Tetas 1 site, located in the Central Plateau of Santa Cruz, Argentina. Regional trends for the period show the practice of knnaping and bifacial reduction techniques, greater artefactual density and the increase in blade production, all these compared to previous moments. The aim is to analyze production strategies followed in the manufacture of stone tools. The assemblage is approached from a techno morphological perspective that pretends to identify relationships between the artifacts, to evaluate knnaping activities, techniques and decisions involved with production processes. The results indicate selection of local raw materials, employment of standardized and non-standardized reduction techniques of cores, increase in choice of blades for the manufacture of artifacts and predominance of production sequences with low work investment.
\end{abstract}

Keywords: Lithic technology; Production strategies; Middle Holocene; Central Plateau; Patagonia.

\section{Introducción}

En el presente artículo estudiamos la tecnología lítica de los grupos que durante el Holoceno medio habitaron la Cueva 1 de Cerro Tres Tetas-C3T1-, en la meseta Central de Santa Cruz, Patagonia Argentina. Esta región reúne testimonios del desarrollo sostenido de sociedades con modo de vida cazador-recolector desde su arribo a fines del Pleistoceno, hasta momentos históricos recientes (Cueto et al, 2017). Las evidencias de actividades humanas en C3T1 durante el Holoceno medio se suman a aquellas registradas en otras ocupaciones contemporáneas de la región. Estas se distribuyen en poco más de una decena de sitios como Los Toldos 2, 3 y 13 (LT2, LT3 y LT13), La Martita 4 (LM4), El Verano (EV), Cueva Maripe (CM), Alero el Puesto 1 (AEP1), La Mesada (LM), Casa del Minero 1 (CDM1), La Ventana (LV), La Lavandería, La Gruta 2 y Viuda Quenzana 8 (Cardich et al, 1993-1994; Cueto et al, 2017; Durán 1990; Franco et al, 2013; Skarbun, 2011).

En relación con la tecnología lítica, los patrones para el Holoceno medio muestran una mayor densidad artefactual que en periodos previos (Hermo y Magnin, 
2012). Se emplean materias primas silíceas locales (e.g. sílex, calcedonia, xilópalo, toba silicificada) de muy buena calidad, y la obsidiana, materia prima alóctona procedente de Pampa del Asador, se utiliza prácticamente en todos los sitios de la meseta y aumenta su representación en los conjuntos alcanzando un promedio de 2,62\%, frente a las proporciones registradas para el Holoceno temprano $(0,82 \%)$ y el Pleistoceno final $(0,33 \%)$ (Cueto et al, 2018; Skarbun, 2011). A su vez, se produce un incremento en la diversidad de clases de herramientas, entre las que predominan los raspadores. En este período se registran las primeras bolas de boleadora, destinadas a la caza. Se evidencia el desempeño de técnicas de talla y de reducción bifacial, aunque se han recuperado pocos instrumentos de factura bifacial en capa, además de las puntas de proyectil (Skarbun, 2011). Este patrón podría vincularse con una práctica de descarte diferencial de estos instrumentos, abandonados fuera de las cuevas debido a cambios en el uso del espacio (Hermo y Magnin 2012). Además se registra un aumento en la producción de láminas entre los productos de la talla, esto surge de aquellos conjuntos que presentan información cuantitativa -LT2 y 13, CDM1, AEP1, CM, LM y LV(Cardich et al, 1993-1994; Cattáneo, 2002; Cueto et al, 2017; Skarbun, 2011; Hermo y Magnin, 2012) como de los que solo se dispone de descripciones cualitativas -LT3, EV y LM4- (Cardich et al, 1973; Duran, 1990). A su vez se incrementa el empleo de láminas como forma base para la manufactura de instrumentos, así en algunos conjuntos -LT2 y 13, CDM1, LM y LV- llega a dominar el empleo de láminas como soporte, o alcanza la misma proporción que las lascas -CM componente inferior-. Mientras en otros -AEP1, CM componente superior- si bien su representación es alta, domina la selección de lascas como forma base (Cardich et al, 1993-1994; Cattáneo, 2002; Cueto et al, 2017; Skarbun, 2011; Hermo y Magnin, 2012).

Los antecedentes permiten delinear una caracterización parcial de la tecnología del período, en base a algunos patrones de los instrumentos y las materias primas. No obstante, es escasa la información sobre núcleos y prácticas involucradas en su explotación, como de las características de los productos de talla y las actividades a las que remiten. Esto se debe a los objetivos que guiaron las investigaciones, orientados a definir secuencias culturales para la región en base a la clasificación tipológica de instrumentos (Cardich et al, 1973), a examinar desde un enfoque tecno-tipológico una sola clase de artefacto (Duran, 1990), o a la presentación integral de un sitio, aproximación en la que habitualmente se efectúa una descripción sucinta de los materiales (Franco et al, 2003). En este artículo el objetivo es analizar las estrategias de producción seguidas en la fabricación de los instrumentos en la unidad 4 del sitio C3T1. Se realiza el abordaje desde una perspectiva tecnomorfólogica orientada a la identificación de relaciones entre los artefactos, con el propósito de evaluar las actividades de talla, las técnicas y las decisiones involucradas en los diversos procesos de producción.

\section{La Cueva 1 de Cerro Tres tetas, paisaje y estudios previos.}

La meseta central de Santa Cruz es una región extensa, con paisajes diversos, que contiene planicies de rodados, valles fluviales, cañadones, serranías rocosas, bajos sin desagues y mesetas volcánicas. Es un paisaje de origen principalmente volcánico con gran disponibilidad de materias primas líticas aptas para la talla, de distribución bastante homogénea (Skarbun, 2011). La Localidad Arqueológica Cerro Tres Tetas se emplaza en la estancia San Rafael. A partir de estudios sistemáticos del paisaje cercano al sitio C3T1 se identificaron fuentes de materias primas silíceas, sectores de densidad alta de material arqueológico en superficie, asociados a vertientes, zanjones y mantos basálticos; y amplios espacios de terreno, bordes de laguna y bajos temporarios que poseen densidad baja (Paunero et al, 2016). La cueva se encuentra sobre un morro que está rodeado por un bajo atravesado por un cauce. Posee una estratigrafía con siete unidades, en la que se identificaron contextos que abarcan desde el Pleistoceno final hasta momentos post conquista. La Unidad 4 asignable al Holoceno medio fue fechada en $5220 \pm 70$ AP (LP-538) a partir de una muestra de carbón (Paunero et al, 2007).

Previamente se efectuó un abordaje del conjunto lítico orientado a evaluar la funcionalidad. Se identificó el empleo de instrumentos confeccionados sobre lascas y láminas -e.g. cuchillos, raederas y raspadores-, evidencias de enmangue de raspadores distales sobre lamina, y el desarrollo de trabajos de corte con láminas de bordes naturales (Paunero et al, 2007). En el estudio se comunican datos generales del conjunto, como las rocas utilizadas, las clases y cantidades de instrumentos y productos de talla -exceptuando los núcleos-, sin evaluar las estrategias de producción. El conjunto posee piezas termoalteradas de las cuales la mayor parte se habría modificado luego de la talla y el descarte en los fogones, por posibles prácticas de mantenimiento del espacio. Mientras un segmento menor sugiere el empleo del tratamiento térmico de núcleos para la producción de cuchillos de sílex (Frank 2016).

\section{Procedimientos metodológicos}

Para el análisis tecno morfológico se siguieron propuestas de descripción y clasificación del ámbito local y regional (Aschero, 1975; Cardich y Flegenheimer, 1978; Cueto, 2015; Paunero y Castro, 2001; Skarbun, 2011). Se caracterizó todo el material lítico, considerando núcleos, productos de la talla y artefactos formatizados, debido a que cada grupo brinda información que permite abordar diferentes prácticas de la producción. Esta aproximación permitió evaluar la variabilidad del conjunto 
a partir de cada atributo morfológico, tecnológico y examinar las características de los artefactos generados durante distintos momentos de la secuencia de producción: preparación/reducción inicial del núcleo (descortezamiento); talla del núcleo y extracción de soportes; formatización final de los artefactos -retalla, retoque y adelgazamiento bifacial- (Cueto et al, 2014; Skarbun, 2011). Los momentos considerados fueron formulados a partir de las propuestas de Collins (198990) y Tixier et al, (1980), de nuestras experimentaciones (Cueto, 2015; Frank 2011) y de investigadores de la meseta que plantearon este tipo de estudios (Mansur, 1984; Cardich et al, 1993-94). La identificación de las litologías se llevó a cabo macroscópicamente, según características de color, brillo, transparencia, textura, inclusiones, corteza y granulometría (Cueto et al, 2018; Skarbun 2011).

\section{Resultados}

El conjunto lítico del componente reúne 1737 artefactos, entre los que se encuentran 15 núcleos, 1592 productos de talla y 47 artefactos formatizados, entre otros (Tabla 1). La materia prima más utilizada es el sílex $(66,27 \%)$, seguido por la calcedonia, el xilópalo, y otras en proporciones inferiores al 6\% (Tabla 1) entre las que destaca la obsidiana con una representación que supera el promedio registrado en ocupaciones contemporáneas para la meseta central. Mientras que las litologías más representadas en el conjunto corresponden a rocas silíceas locales, de calidad muy buena a excelente, de matriz homogénea, que no poseen grietas e inclusiones o contienen pocas y diminutas.

Entre los núcleos predominan los de sílex. El 53,33\% de estas piezas posee forma poliédrica, también hay discoidales (20\%), prismáticos (13,33\%), y bifacial y piramidal con un 6,67\% respectivamente. Mayormente presentan tamaño mediano y grande, seguidos de otros de tamaño chico y muy grande (Figura 1a). La mayoría se encuentran agotados (60\%) y no poseen corteza o registran un remanente de escasa a parcial, mientras el resto se encuentran activos y retienen corteza -parcial a abundante-. De estos núcleos se extrajeron lascas (73,34\%), láminas y laminillas (20\%) o la combinación de las anteriores $(6,66 \%)$; y registran entre 5 y 22 extracciones (Figura 2b y c).

En cuanto a los productos de la talla prevalecen ampliamente los de sílex $(65,64 \%)$, sobre los de calcedonia, xilópalo y otras rocas de procedencia local y alóctona (Tabla 1). Entre estos hay 648 fragmentos de talla o piezas indeterminables, asignables a las distintas litologías con tamaños muy chico y chico. En relación con la clase de productos, el 89,62\% corresponde a lascas, en menor representación hay láminas, seguidas de otras (Figura 3a). El estado de los productos evidencia una alta frecuencia de lascas enteras $(67,38 \%)$, seguidas por lascas fracturadas sin talón y en menor proporción con talón (Figura 3b). El 23,62\% de estas piezas presenta remanente de corteza en cara dorsal, mientras que el resto no posee. Para estimar las variables dimensionales se tomaron en cuenta los productos de la talla enteros y fragmentados con talón cuya fractura no haya afectado las dimensiones consideradas (largo, ancho). Los tamaños mejor representados en la muestra son muy chico $(61,48 \%)$ y chico, siendo escasos los soportes de tamaños mayores (Figura 1b). Con relación al módulo largo/ancho de los productos dominan el medio (44,87\%) y el corto $(39,14 \%)$, sobre el largo; y respecto al módulo ancho/espesor domina el medio (52,34\%), seguido por el delgado $(33,58 \%)$ y el grueso. El conjunto está compuesto

Tabla 1. Clases de artefactos por materia prima. Prod. Producto; Frg. Fragmento.

Table 1. Classes of raw material artifacts. Prod. Product; Frg. Fragment.

\begin{tabular}{|c|c|c|c|c|c|c|c|c|c|c|}
\hline & $\frac{\stackrel{x}{\leftrightarrows}}{\dot{\omega}}$ & $\frac{\dot{8}}{\frac{0}{\pi}}$ & $\begin{array}{l}\frac{0}{\bar{N}} \\
\frac{0}{0} \\
\frac{\bar{x}}{x}\end{array}$ & 造 & $\frac{.0}{0}$ & 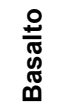 & $\begin{array}{l}\text { 을 } \\
\text { 틀 } \\
\text { 흐 }\end{array}$ & 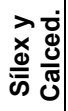 & 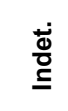 & 픈 흉 \\
\hline Núcleo & 12 & 2 & . & . & . & . & . & 1 & . & $15 / 0,88$ \\
\hline $\begin{array}{l}\text { Prod. } \\
\text { detalla }\end{array}$ & 1045 & 241 & 171 & 80 & 50 & 1 & . & 1 & 3 & $1592 / 91,66$ \\
\hline $\begin{array}{c}\text { Artefacto } \\
\text { formatizado }\end{array}$ & 35 & 6 & 4 & 1 & 1 & . & . & . & . & $47 / 2,7$ \\
\hline Preforma & 1 & . & . & . & . & . & . & . & . & $1 / 0,05$ \\
\hline Bifaz & 1 & . & . & . & . & . & . & . & . & $1 / 0,05$ \\
\hline $\begin{array}{l}\text { Prod. } \\
\text { técnico }\end{array}$ & . & . & . & . & . & 1 & . & . & . & $1 / 0,05$ \\
\hline $\begin{array}{c}\text { Frg. } \\
\text { estallido } \\
\text { térmico }\end{array}$ & 49 & 3 & 1 & 8 & . & . & . & . & . & $61 / 3,52$ \\
\hline Frg.indet. & 8 & 2 & 1 & 4 & . & . & 4 & . & . & 19 / 1,09 \\
\hline $\begin{array}{c}\text { Total } \\
\text { materia } \\
\text { prima n / \% }\end{array}$ & $\begin{array}{l}1151 / \\
66,27\end{array}$ & $\begin{array}{c}254 / \\
14,6 \\
2 \\
\end{array}$ & $\begin{array}{c}177 / \\
10,1 \\
9\end{array}$ & $\begin{array}{l}93 / \\
5,35\end{array}$ & $\begin{array}{c}51 / \\
2,9 \\
4 \\
\end{array}$ & $\begin{array}{c}2 / \\
0,1 \\
1\end{array}$ & $\begin{array}{c}4 / \\
0,2 \\
4 \\
\end{array}$ & $\begin{array}{c}2 / \\
0,1 \\
1\end{array}$ & $\begin{array}{c}3 / \\
0,17\end{array}$ & $1737 / 100$ \\
\hline
\end{tabular}


principalmente por piezas originadas durante la actividad de talla del núcleo $(56,27 \%)$, seguido de lascas asignables a la formalización final de los instrumentos -predominio de retoque y retalla- y evidencias del descortezamiento de núcleos (Figura 3c). Además, se recuperó una pieza de basalto de tamaño chico y corteza abundante, clasificada como producto técnico, originada de la fractura por fatiga de uso de la porción activa de un percutor.

Los artefactos formatizados se agrupan en ocho clases, entre las que dominan los cuchillos $(n=10)$, seguidos por raederas, raspadores y lascas retocadas, entre otras de frecuencias inferiores (Figura $2 a$ y c). El 51,07\% de los artefactos se conserva entero, sobre un $46,81 \%$ fragmentado y un $2,12 \%$ indeterminado. Predominan los instrumentos de tamaño mediano $(45,45 \%)$, sobre los de dimensión chico $(27,28 \%)$, grande $(24,24 \%)$ y muy grande (3,03\%). Registran módulos de largo/ancho mayoritariamente largo (51,52\%), seguido de medio $(45,45 \%)$ y corto $(3,03 \%)$; y módulos de ancho/espesor principalmente medio $(66,67 \%)$ seguido de grueso $(27,27 \%)$ y delgado $(6,06 \%)$. Todos los artefactos son unifaciales, y como forma base para su manufactura se han empleado lascas $(51,07 \%)$, una alta representación de láminas $(42,55 \%)$ y un $6,38 \%$ indeterminado. El $74,47 \%$ de los artefactos no presenta corteza, mientras que el resto posee algún remanente.

Los instrumentos registran 77 filos formatizados, la mayoría posee un filo (51,07\%), el 36,18\% tiene dos, el $10,63 \%$ posee tres, y el $2,12 \%$ ostenta cuatro. Prácticamente en todas las clases de instrumentos dominan los filos largos, de ángulos agudos, y solo los filos de raspador registran a su vez ángulos abruptos. Los filos están regularizados principalmente por retoque, seguidos de microrretoque, su combinación y otros (Tabla 2), procedimientos efectuados por percusión y de manera muy escasa por presión. El 96,2\% posee modificación continua y el resto discontinua, por lascados directos $(97,42 \%)$ excepto un caso de trabajo inverso y otro bifacial. En cuanto a la profundidad de los lascados, predominan los de tipo ultramarginal, mientras la forma de los mismos es casi exclusivamente escamosa, principalmente de tipo irregular (Tabla 2).

\section{Discusión y conclusiones}

Los grupos que habitaron C3T1 seleccionaron materias primas silíceas de fuentes locales, para la manufactura de los artefactos. En este sentido las decisiones vinculadas al abastecimiento de las rocas son similares a las estrategias implementadas en las ocupaciones contemporáneas de la región, y se relacionan con la elevada disponibilidad y calidad del recurso en la meseta. Además, emplearon obsidiana como en casi todos los sitios de la meseta y en mayor proporción que en periodos previos, como señalan las tendencias (Cueto et al. 2018).

Las evidencias indican el ingreso al sitio de núcleos de sílex y calcedonia con reserva de corteza, también de xilópalo como sugiere la presencia de lascas de preparación de plataformas de percusión, y posiblemente nódulos pequeños de obsidiana. Esta práctica es consistente con los restos que evidencian tareas de descortezamiento, aunque en baja proporción, dentro de la cueva. Una vez agotados, la mayor parte de los núcleos fueron descartados en este espacio. A su vez, otros núcleos de sílex, calcedonia y toba silicificada, incluso aquellos prismáticos y piramidales vinculados a la producción de láminas, habrían sido descortezados y preparados fuera del sitio. Luego podrían haber ingresado en formato de núcleo, de formas base y de preformas, acorde con las tendencias regionales para áreas de alta disponibilidad de rocas. En el sitio se efectuaron principalmente actividades de talla y obtención de soportes a partir de núcleos no estandarizados -poliédricos y discoidalesgenerando mayormente lascas y en ocasiones lascas y soportes laminares -lascas largas y láminas-. Mientras, el principal aporte de láminas al conjunto provendría de la explotación de los núcleos prismáticos y piramidales
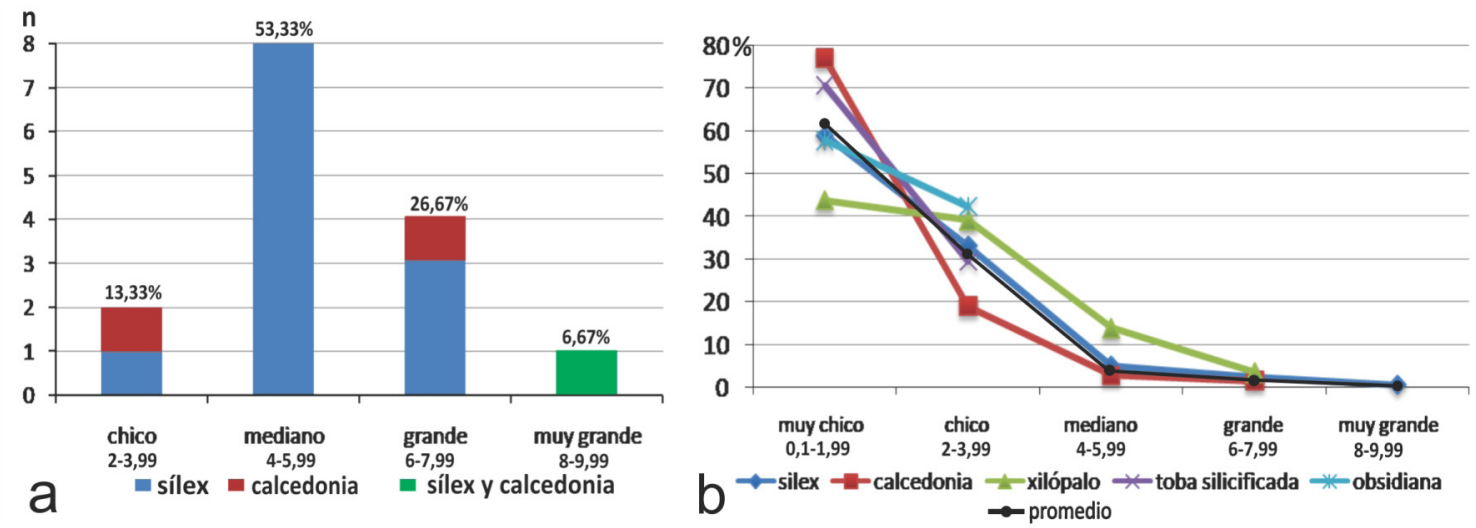

Figura 1. a. Tamaño de núcleos según materia prima. b. Tamaño de productos de talla según materia prima.

Figure 1. a. Size cores as raw material. b. Size flakes as raw material. 


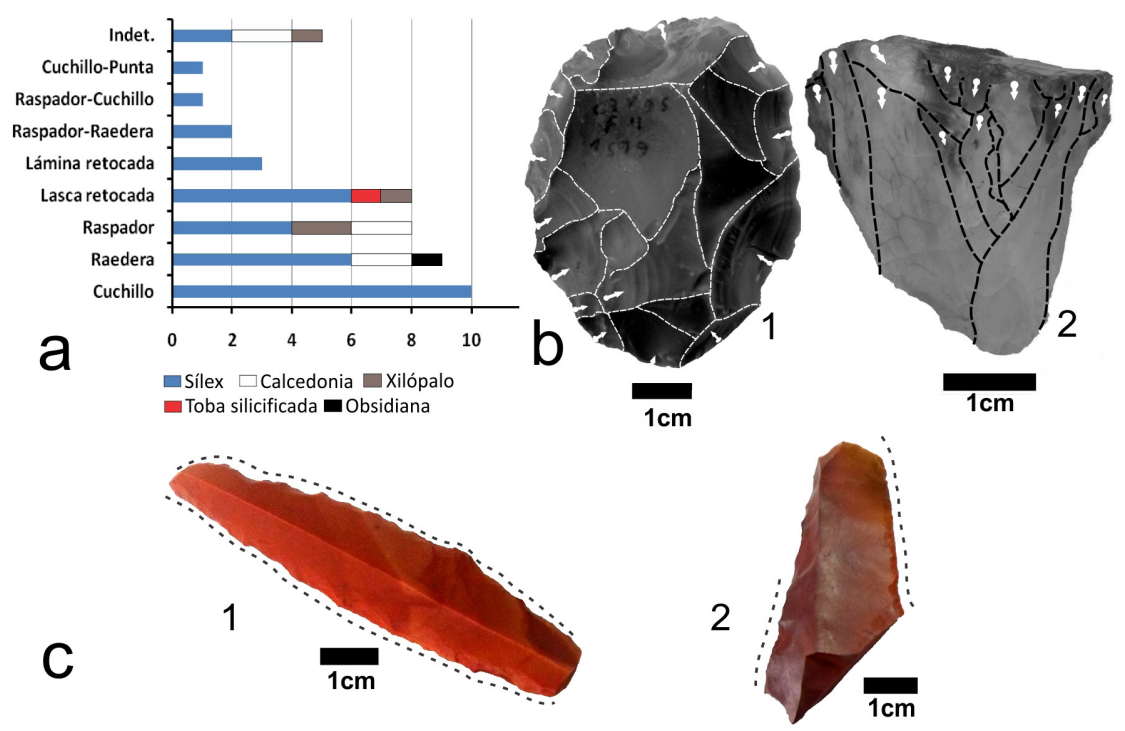

Figura 2. a. Frecuencia de artefactos formatizados según materia prima. b. Núcleos. 1. Bifacial, de sílex, negativos de lasca. 2. Prismático, de calcedonia, negativos de lámina. c. 1. Raedera y 2 . Cuchillo. Ambos de sílex y con dos filos.

Figure 2. a. Frequency of formal tolos as raw material. b. Cores. 1. Bifacial, of flint, flake negatives. 2. Prismatic, of chalcedony, blade negatives. c. 1. Side scraper and 2. Knife. Both of flint and with two edges.

-de sílex y de calcedonia- que implicaron mayor trabajo en su preparación y que fueron abandonados una vez agotados, dentro de la cueva.

Para la confección de los artefactos formatizados predomina la selección de lascas como soporte, y si bien la representación de las láminas es menor entre los productos de la talla del conjunto se evidencia un incremento considerable en la elección de estos soportes -de tamaño mediano y superior- para emplear como forma base, respecto a periodos previos. Estas prácticas resultan consistentes con las desarrolladas en el sitio Alero el Puesto 1 -CM componente superior-y a su vez se enmarcan en la tendencia al incremento por el empleo de láminas como forma base de los instrumentos, registrada para la meseta.

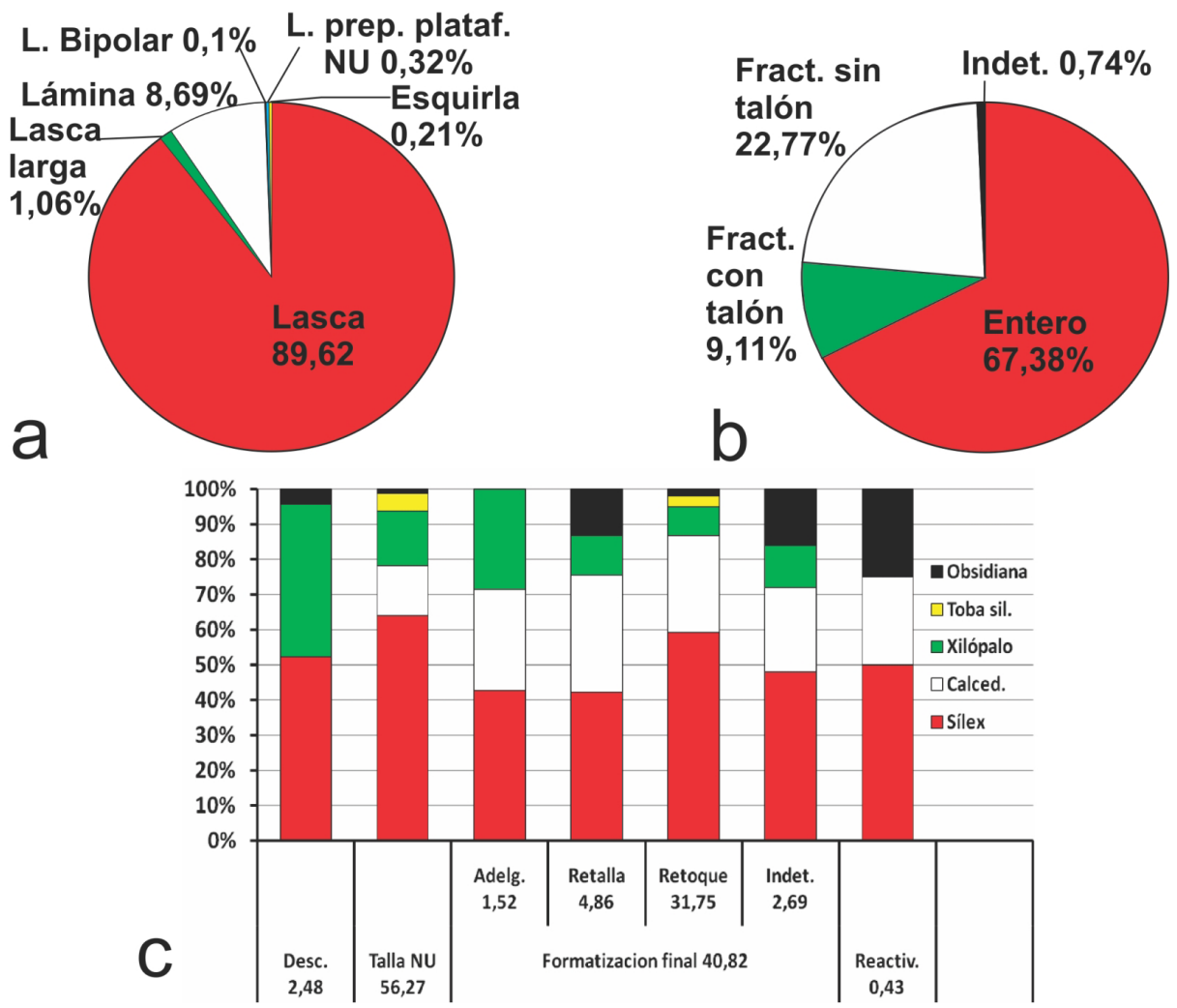

Figura 3. Productos de talla a. Clase. b. Estado. c. Productos según materia prima y momento de la secuencia de producción.

Figure 3. Debitage a. Type. b. State. c. Flakes according raw material and steps of production process. 
Tabla 2. Caracteres del filo (\%). a.o. agudo oblicuo; a.m.o. agudo muy oblicuo; ab.m.o. abrupto muy oblicuo; ab.o. abrupto oblicuo; R. retoque; M. microrretoque; T. talla; U. ultramarginal; M. marginal; I. irregular; R. regular; E. escalonado; PL. paralelo laminar.

Table 2. Edge characterss (\%). a.o. acute oblique; a.m.o. acute very oblique; ab.m.o. abrupt very oblique; ab.o. abrupt oblique; R. retouch; M: micro-retouch; T. knapping; U. ultramarginal; M. marginal; I. irregular; R. regular; E: stepped; PL: blade parallel.

\begin{tabular}{|c|c|c|c|c|c|}
\hline $\begin{array}{l}\text { Clase I } \\
\text { filos }\end{array}$ & Extensión & Ángulo & Ancho & Profundidad & Forma \\
\hline $\begin{array}{l}\text { Cuchillo } \\
\text { / } 15\end{array}$ & $\begin{array}{l}\text { largo } 60 \\
\text { corto } 40\end{array}$ & $\begin{array}{c}\text { a.o. } 66,67 ; \\
\text { a.m.o. } 33,33\end{array}$ & $\begin{array}{c}\text { R 53,34, } \\
\text { M 20; } \\
\text { RyM 20; } \\
\text { RyT 6,66 }\end{array}$ & $\begin{array}{c}\text { U 86,66; } \\
\text { M 6,67; } \\
\text { MyU 6,67 }\end{array}$ & $\begin{array}{l}\text { I } 80 \\
\text { R } 20\end{array}$ \\
\hline $\begin{array}{c}\text { Raedera } \\
\text { / } 17\end{array}$ & $\begin{array}{l}\text { largo } \\
52,95 \\
\text { corto } \\
47,05\end{array}$ & $\begin{array}{c}\text { a.m.o. } 64,71 ; \\
\text { a.o. } 29,41 ; \\
\text { ab.m.o. } 5,88\end{array}$ & $\begin{array}{c}\text { R 76,48; } \\
\text { RyM 17,64; } \\
\text { RyT 5,88 }\end{array}$ & $\begin{array}{l}\text { U 64,7; } \\
\text { M 35,29 }\end{array}$ & $\begin{array}{c}\text { I 94,11; } \\
\text { R 5,88 }\end{array}$ \\
\hline $\begin{array}{l}\text { Raspador } \\
\quad / 15\end{array}$ & $\begin{array}{c}\text { largo } \\
53,85 \\
\text { corto } \\
38,46 \text { ext. } \\
7,69\end{array}$ & $\begin{array}{l}\text { a.m.o. 30,76; } \\
\text { a.o., ab.o. y } \\
\text { ab.recto 23,08 } \\
\quad \text { (c/u) }\end{array}$ & $\begin{array}{l}\text { RyM 53,85; } \\
\text { R 38,46; } \\
\text { MyT 7,69 }\end{array}$ & $\begin{array}{l}\text { U 61,53; } \\
\text { M 23,07; } \\
\text { MyU 7,69; } \\
\text { Indet. 7,69 }\end{array}$ & $\begin{array}{c}\text { I 84,61; } \\
\text { E y PL } \\
7,69 \\
\text { (c/u) }\end{array}$ \\
\hline $\begin{array}{c}\text { Lasca } \\
\text { retocada / } \\
8\end{array}$ & $\begin{array}{l}\text { corto } 62,5 \\
\text { largo } 37,5\end{array}$ & $\begin{array}{l}\text { a.o. } 62,5 ; \\
\text { a.m.o. } 37,5\end{array}$ & $\begin{array}{l}\text { RyM 50; } \\
\text { R 37,5; } \\
\text { RyT 12,5 }\end{array}$ & U 100 & $\begin{array}{l}\text { I } 75 \\
\text { R } 25\end{array}$ \\
\hline $\begin{array}{c}\text { Lámina } \\
\text { retocada / } \\
3\end{array}$ & largo 100 & $\begin{array}{c}\text { a.o. } 66,67 ; \\
\text { a.m.o. } 33,33\end{array}$ & $\begin{array}{c}\text { M, R y R y } \\
\text { M } 33,33 \\
(\mathrm{c} / \mathrm{u})\end{array}$ & U 100 & 100 \\
\hline Otros / 19 & $\begin{array}{l}\text { largo } \\
52,63 \\
\text { corto } \\
47,36\end{array}$ & $\begin{array}{c}\text { a.m.o. } 63,15 ; \\
\text { a.o. } 26,31 ; \\
\text { a.rasante y } \\
\text { ab.o. } 5,27 \\
\text { (c/u) }\end{array}$ & $\begin{array}{c}\text { R 47,37; } \\
\text { RyM y RyT } \\
\text { 21,05 (c/u); } \\
\text { M 10,53 }\end{array}$ & $\begin{array}{l}\text { U y M } 42,1 \\
\text { (c/u); } \\
\text { MyU 10,53; } \\
\text { M 5,27 }\end{array}$ & $\begin{array}{l}\text { I 89,48; } \\
\text { R 10,52 }\end{array}$ \\
\hline
\end{tabular}

Empleando los soportes seleccionados, de tipo lasca y de modulo laminar -lámina y lasca larga-, los talladores confeccionaron las distintas clases de instrumentos que dominan el conjunto (cuchillos, raspadores y raederas), mientras que para la manufactura de algunas clases (raspador-cuchillo, raspador-raedera, y lámina retocada) solo escogieron láminas, y para otras (lascas retocadas y cuchillo-punta) se valieron de lascas únicamente. La configuración de estos instrumentos se realizó mediante procedimientos técnicos que involucran la modificación unifacial por percusión y la frecuente regularización de más de un borde, casi exclusivamente por percusión.

Las tareas de formatización final se caracterizan por la modificación continua directa ultramarginal de los bordes por retoque, microrretoque o su combinación, de tipo escamoso irregular. De este modo se diseñaron filos mayormente largos de ángulos agudos. Los procedimientos identificados remiten principalmente al desarrollo de una estrategia de producción expeditiva o que requiere de poca inversión de trabajo en la manufactura de estos instrumentos. Sin embargo, en el caso de los raspadores elaborados sobre lámina, la secuencia habría requerido de mayor inversión de trabajo debido a la confección e incorporación del mango. A su vez, es posible que se hayan seguido otras estrategias que sugieren mayor inversión de trabajo en la manufactura. Una de ellas destinada a fabricar dos cuchillos y un artefacto indeterminado, que implicó el desarrollo de la técnica de tratamiento térmico sobre núcleos de sílex, a partir de los cuales se obtuvieron los soportes laminares mediante procedimientos que implican mayor control debido a la menor dureza y al incremento de la fragilidad provocada en la roca por la termoalteración. La otra sugiere el desarrollo de la técnica de reducción bifacial para la producción de un bifaz de sílex de tamaño mediano, evidenciada a su vez por algunas lascas de adelgazamiento recuperadas en el conjunto.

Consideramos que en la Unidad 4 se articula una serie de decisiones y prácticas tecnológicas, que orientan las secuencias hacia la producción de un equipo de artefactos entre los cuales los soportes que requieren mayor predeterminación y los diseños elaborados sobre módulos laminares revisten renovada importancia para los grupos, respecto a las ocupaciones previas de la meseta. Este cambio podría vincularse a la necesidad por parte de los artesanos de lograr un aprovechamiento distinto de las rocas generando soportes laminares con más de un filo natural que pudieran usarse de manera directa, tal como se ha identificado para trabajos de corte de diversas sustancias dentro del conjunto (Paunero et al. 2007). A su vez, estos soportes podrían haberse procurado ya que habitualmente brindan la posibilidad de regularizar más de un borde, hecho que se evidencia prácticamente en la mitad de los artefactos formatizados de la unidad, y en consecuencia podría incrementar las 
posibilidades de uso de los instrumentos en una misma o en diferentes acciones de trabajo. Además, es posible que el diseño laminar de algunos artefactos empleados en determinadas labores demandaran un cambio en la forma de prensión tornándose necesario su enmangue, práctica que también ha sido sugerida para otros contextos en la meseta (Duran 1990; Mansur 1984). Evidencias de este tipo se han registrado en raspadores empleados para el curtido de pieles en el conjunto de la unidad 4 (Paunero et al, 2007), y en raspadores destinados a raspar/pulir elementos óseos en LT13 (Cueto et al, 2017). Además, tanto la estandarización en la producción de los diseños laminares como las distintas posibilidades que ofrecen a nivel funcional, en especial para raspadores y cuchillos, han sido convalidadas y resaltadas para el conjunto contemporáneo de LT13 (Cueto et al, 2017).

Haber indagado sobre la tecnología de esta ocupación, poniéndola en dialogo con los patrones regionales propuestos hasta el momento, nos permitió profundizar la comprensión de sus características particulares, las preferencias y las estrategias de producción de artefactos. A su vez nos posibilita retomar el debate sobre las prácticas tecnológicas en la meseta, y lo actualiza integrando nueva información de C3T1 que permitirá abordar a futuro aspectos sociales y económicos de los grupos con más detalle.

\section{Agradecimientos}

Agradecemos a Shilo Hocsman, María del Pilar Babot y Patricia Escola editores de este volumen y a los evaluadores anónimos por sus valiosos aportes y sugerencias. El trabajo se desarrollo en el marco del proyecto SeCyT/UNLP (PI-N810) dirigido por Rafael Paunero, y del proyecto PPID/UNLP (N033) Paisajes arqueológicos y tecnología de los habitantes de la Meseta Central, la costa atlántica de Santa Cruz y su área intermedia durante el Holoceno Medio, dirigido por Manuel Cueto.

\section{Bibliografía}

Aschero, C. (1975). Ensayo para una clasificación morfológica de artefactos líticos aplicado a estudios tipológicos-comparativos. Argentina, Informe CONICET, Ms.

Cardich, A., Cardich, L. y Hajduk, A. (1973). Secuencia arqueológica y cronología radiocarbónica de la Cueva 3 de Los Toldos (Santa Cruz, Argentina). Relaciones de la Sociedad Argentina de Antropología, VII, 85-123.

Cardich, A. y Flegenheimer, N. (1978). Descripción y tipología de las industrias más antiguas de Los Toldos. Relaciones de la Sociedad Argentina de Antropología. XII, 225-242.
Cardich, A., Paunero, R. S. y Castro, A. S. (1993-1994). Análisis de los conjuntos líticos de la cueva 2 de Los Toldos (Santa Cruz, Argentina). Anales del Instituto de la Patagonia, 22, 149-173.

Cattáneo, G. (2002). Una aproximación a la organización de la tecnología lítica entre los cazadores recolectores del Holoceno Medio/Pleistoceno Final en la Patagonia Austral, Argentina (Tesis doctoral). Facultad de Ciencias Naturales y Museo Universidad Nacional de La Plata. La Plata.

Collins, S. (1989-90). Una propuesta conductual para el estudio de la arqueología lítica. Etnía (3435), 47-65.

Cueto, M. (2015). Análisis de los procesos de uso de artefactos líticos en sociedades cazadorasrecolectoras. Ocupaciones correspondientes a la transición Pleistoceno/Holoceno. Meseta Central de Santa Cruz. British Archaeological Reports. Int. Series 2776. United Kingdom.

Cueto, M., Skarbun, F. y Frank, A. (2014). Tecnología lítica de los cazadores-recolectores de la meseta central patagónica. Balances y perspectivas para una propuesta de integración. En A. Lourdeau, S. Viana y M. Rodet (Ed.).Indústrias líticas na América do Sul: Abordagens Teóricas e Metodológicas. (pp.173-202). Recife: EdUFPE

Cueto, M., Frank, A. y Castro A. (2017). A technomorphological and functional study of late Pleistocene and middle Holocene lithic assemblages from Patagonia Argentina. Quaternary International, (442) 67-79.

Cueto, M., Frank, A. y Skarbun, F. (2018) Explotación de obsidianas en la meseta central de Santa Cruz. Estrategias de producción, uso y circulación. Chungara, 50 (2), 235253.

Duran, V. (1990). Estudio tecno-tipológico de los raspadores del sitio El Verano. Cueva 1. Área La Martita. Santa Cruz. Anales de Arqueología y Etnología, 41/42, 129-163.

Franco, N., Ambrústolo, A., Acevedo, A., Cirigliano, N. y Vommaro, M. (2013). Prospecciones en el sur del macizo del Deseado (Pcia. Santa Cruz, Argentina). Los casos de La Gruta y Viuda Quenzana. En Zangrando A, Barberena R, Gil A, Neme G, Giardina M, Luna L, Otaola C, Paulides S, Salgan, L, Tivoli A. (Ed.), Tendencias teóricometodológicas y casos de estudio en la arqueología de la Patagonia (pp.371-378), Buenos Aires, Argentina.

Hermo, D. y Magnin, L. (2012). Blade and bifacial 
technology in Mid-Holocene occupations at Deseado Massif, Santa Cruz province, Argentina. Quaternary International, (256) 71-77.

Mansur, M.E. (1984). Prehistorie de Patagonie: L'industrie "Nivel 11" Tecnologie lithique et traces d'utilisation. British Archaeological Reports. United Kingdom.

Paunero, R. y Castro, A. (2001). Análisis lítico y funcionalidad del componente inferior de Sitio Cueva 1, Localidad Arqueológica Cerro Tres Tetas, Provincia de Santa Cruz, Argentina. Anales del Instituto de la Patagonia. (29) 189-206.

Paunero, R., Castro, A. y Reyes, M. (2007). Estudios líticos del componente medio del sitio Cueva 1 de Cerro Tres Tetas, Santa Cruz, Argentina. Implicaciones para construir patrones de distribución artefactual y uso del microespacio. En Arqueología de Fuego Patagonia.
Levantando piedras, desenterrando huesos y develando arcanos: 613-622.

Paunero, R., Skarbun, F., Oronó, M., Valiza Davis, C. y Frank, A. (2016). Paisaje Arqueológico en la Meseta Central de Santa Cruz. Análisis Distribucionales en Cerro Tres Tetas. En Actas del XIX Congreso Nacional de Arqueología Argentina. Serie Monográfica y Didáctica. Vol.54. 777-779. Universidad Nacional de Tucumán.

Skarbun, F. (2011). La organización tecnológica en grupos cazadores recolectores desde las ocupaciones del Pleistoceno final al Holoceno tardío, en la Meseta Central de Santa Cruz, Patagonia. BAR International Series 2307. Archaeopress, Oxford.

Tixier, J., Inizan, M., Roche, H. (1980). Prehistorie de la Pierre taille. Circle de Rechearches et d'etudes prehistoriques. France. 